\title{
Cascaded Energy Transfer for Efficient Broad-Band Pumping of High-Quality, Micro-Lasers
}

\author{
C. Rotschild,* M. Tomes, H. Mendoza, T. L. Andrew, T. M. Swager, T. Carmon, \\ and M. A. Baldo
}

\begin{abstract}
Many on-chip optical applications, including spectroscopy, ${ }^{[1]}$ sensing, ${ }^{[2,3]}$ nonlinear optics, ${ }^{[4-6]}$ and optical communications ${ }^{[7,8]}$ require high-finesse, high-quality factor (high-Q) micro-lasers. Such lasers must be exceptionally transparent at their emission wavelength. But if high- $Q$ micro lasers exhibit correspondingly weak absorption at the pump wavelengths, they are challenging to excite. Here, we demonstrate micro-ring lasers that exhibit $Q>5.2 \times 10^{6}$ and a finesse of $>1.8 \times 10^{4}$ with a direct-illumination, non-resonant pump. The micro-rings are coated with a combination of three organic dyes. This cascaded combination of near and ultimately far field energy transfer reduces materiallosses by a factor of more than $10^{4}$, transforming incoherent light to coherent light with high quantum-efficiency. The operating principle established here is general and can enable fully integrated on-chip, high finesse micro lasers without the complications of coupled pump and emitter resonators.

The quality factor of a lasing cavity is the ratio between the stored power and the power dissipated by the cavity. ${ }^{[9]}$ High$Q$ lasers exhibit quality factors of $>10^{6}$, equivalent to photon cavity propagation lengths beyond one meter. Thus, in microresonators, a high $Q$-factor implies many photon cycles in the cavity and a high finesse. Such a laser must be based on a low-loss resonator, such as an on-chip toroidal resonator with nearly atomically-perfect surfaces. ${ }^{[10]}$ Silica-based high- $Q$ resonators are particularly attractive, because they are constructed on silicon chips. But there is presently no cost effective way to integrate the optical gain element for a high- $Q$ source within the silica-on-silicon based architecture. To date, high- $Q$ optical sources have required highly absorptive, low- $Q$ gain media
\end{abstract}

Dr. C. Rotschild, H. Mendoza, Prof. M. A. Baldo

Department of Electrical Engineering and Computer Science

Massachusetts Institute of Technology

77 Massachusetts Avenue, Cambridge, MA 02139, USA

E-mail: carmelr@mit.edu

Dr. C. Rotschild

Department of Mechanical Engineering

Technion - Israel Institute of Technology

Haifa 32000, Israel

M. Tomes, Dr. T. Carmon

Department of Electrical Engineering and Computer Science

University of Michigan

Ann Arbor, Michigan, 48109, USA

T. L. Andrew, Prof. T. M. Swager

Department of Chemistry

Massachusetts Institute of Technology

77 Massachusetts Avenue, Cambridge, Massachusetts 02139, USA

DOI: $10.1002 / \mathrm{adma} .201100467$ based on III-V semiconductors injection locked to a high- $Q$ micro resonator. ${ }^{[11,12]}$ Instead, it may be preferable to integrate the gain media directly into a silica-based micro resonator. This could enable arrays of high- $Q$ lasers pumped without optical feedback using an off-chip broadband source, such as a lightemitting diode.

To develop a low-cost solution for silicon-compatible on-chip high-Q optical sources, we coat silica resonators with a combination of organic dyes. The materials are selected to demonstrate cascaded energy transfer, which concentrates the optical pump and overcomes the mismatch in the spectrally and spatially broad pump and the narrow modes of the high-finesse resonant cavity. The broadband pump excites a short wavelength dye in a low $Q$-factor cavity, thereby avoiding the need to resonantly match the pump. Next, the low $Q$-factor cavity pumps a longer wavelength dye in what is effectively a higher $Q$-factor cavity, and the process can be repeated, until the desired $Q$-factor is met. All cavities are on the same resonator and differ only in the wavelength of their operation. Detuning of one cavity with respect to the other cannot occur, eliminating the instability normally associated with resonant pumping of high-Q lasers. ${ }^{[13]}$ The reduction in étendue as the coherence increases during the energy cascade is not achieved at the cost of a lower quantum efficiency. Rather, each dye re-emits the pump energy into a successively smaller étendue, at the cost of heat dissipation. ${ }^{[14]}$ The result is a pumping technique for high-finesse lasers that is independent of the coherence of the pump source. In contrast to conventional broad band pumping techniques, the method has high quantum efficiency, which is reflected in a low power threshold.

Figure 1a shows the micro-ring resonator used in this work. As shown in Figure 1b, the resonators are coated with three dyes; AlQ3, DCJTB(1\%), and Terrylene ${ }^{[15]}$ (see Methods). The concentration of the terminal dye, Terrylene is varied between $0.0075 \%$ and $0.02 \%$ by weight. A numerical simulation ${ }^{[16]}$ of the lasing mode is presented in Figure 1c. The lasing whispering gallery mode is detected via its residual forward scattering (Figure 1)d.

As described in Figure 2a, the absorption and emission spectra of the three dyes form an energetic cascade. Each dye is chosen such that its emission (Figure 2b), matches the absorption peak of the next material (Figure 2c). The pump is absorbed by the AlQ3 which transfers its energy to DCJTB via Förster (near field) energy transfer at an efficiency close to $100 \%,{ }^{[17-19]}$ Due to the large Stokes shift, DCJTB emits at around $\lambda=650 \mathrm{~nm}$, where the cavity is very transparent, and where, neglecting any excited state absorption, the $Q$-factor of the DCJTB laser is expected to be $Q \sim 5 \times 10^{5}$. 
(a)

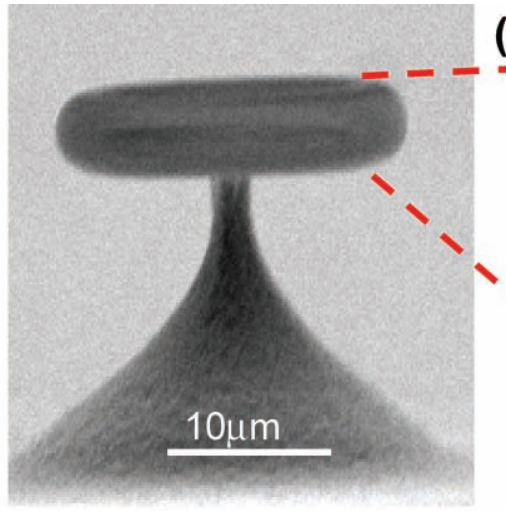

(c)

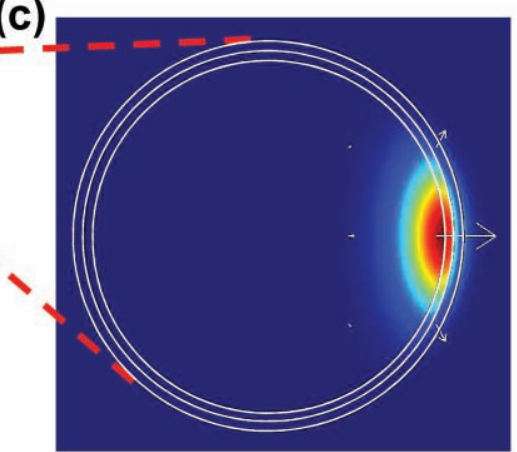

(b)

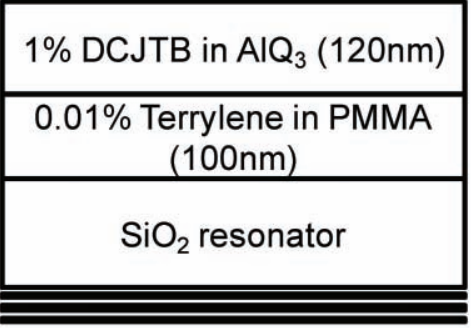

(d)

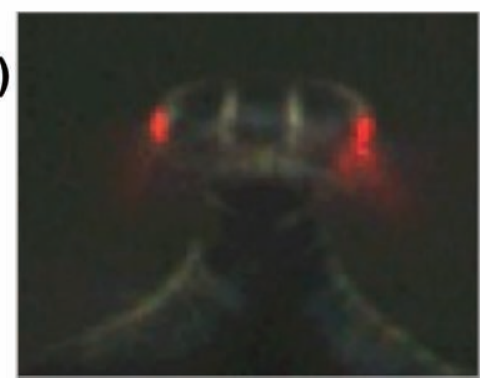

Figure 1. (a) A typical micro-ring resonator. (b) The structure of the gain materials deposited on the resonator. (c) Numerical simulation of the lasing mode with polarization indicated by the arrows, and (d), a typical example of lasing.

Consequently, the DCJTB emission can propagate for large distances in the cavity, until it is absorbed by the final dye in the cascade, Terrylene, which lases at $\lambda=670-700 \mathrm{~nm}$. At these wavelengths the device is transparent enough to support a $Q$-factor over $10^{6}$ (black line). The upper curve in Figure $2 \mathrm{~b}$ (pink line) shows the observed multi-line lasing spectra for a $2 \mathrm{~mW} \mu \mathrm{m}^{-2}$ pump intensity. Cavities that lacked $\mathrm{AlQ}_{3}$ and DCJTB did not exhibit lasing due to the absence of an energy cascade.

Figure 3 shows the evolution of the lasing spectrum with pump power (Figure 3a) and also the threshold characteristic (Figure 3b). Between $\lambda=550-650 \mathrm{~nm}$ we observe spontaneous photoluminescence (PL) primarily from DCJTB. There is an artifact of the pump laser at $\lambda=698 \mathrm{~nm}$, which also serves as a monitor of the pump power. Above a pump threshold of $30 \mu \mathrm{W} \mu \mathrm{m}^{-2}$, a sharp peak attributed to Terrylene lasing at about $\lambda=692 \mathrm{~nm}$ rises at a rate 150 times faster than the spontaneous background. There is no evidence of the Purcell effect below threshold because the pump predominantly excites low$Q$ modes spread over the face of the micro-ring, rather than the high- $Q$ modes at the circumference. Indeed, it should be possible to reduce the threshold of this laser significantly by improving the overlap between the pump and the lasing mode, which is currently far below $1 \%$. Another way to improve the process is to optimize the thickness of the layers, which affects the radiative energy transfer and self-absorption of DCJTB and Terrylene. Increasing the pump power produces multi-peak excitation and modal competition, which affect the gain for the major $\lambda=692 \mathrm{~nm}$ line above $1 \mathrm{~mW} \mu \mathrm{m}^{-2}$. We also observe lasing of the intermediate dye (DCJTB) at $\lambda=630 \mathrm{~nm}$ above pump intensities of $1.2 \mathrm{~mW} \mu \mathrm{m}^{-2}$. Lasing in DCJTB may

improve the pumping efficiency of Terrylene due to improved optical confinement of the DCJTB pump.

Next, we measure the $Q$-factor at the Terrylene lasing wavelengths. We determine $Q$ from the photon lifetime in the cavity: $Q=$ $2 \pi \tau v$, where $\tau$ is the photon lifetime in the cavity, and $v$ is the optical frequency. The energy cascade is a multi-event process, with components including the UV pump pulse-duration, DCJTB excited state lifetime, DCJTB photon lifetime, Terrylene excited state lifetime, and Terrylene photon lifetime. To isloate the Terrylene cavity photon lifetime from other dynamics in the laser, we measured the difference in the lasing transient due to a change of the Terrylene concentration. Experimentally, we changed the Terrylene concentration from $0.02 \%$ to $0.0075 \%$, and measured the emission lifetime versus wavelength using a streak camera. Figure 4 shows typical streak camera results taken from nine different resonators at high pump intensity $\left(3.3 \mathrm{~mW} \mu \mathrm{m}^{-2}\right.$ ) to generate sufficient signal. The multi-line spectral response in the (a)

(b)

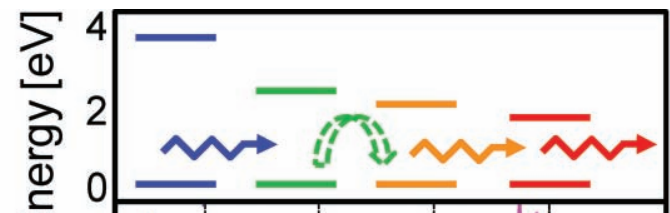

(c)

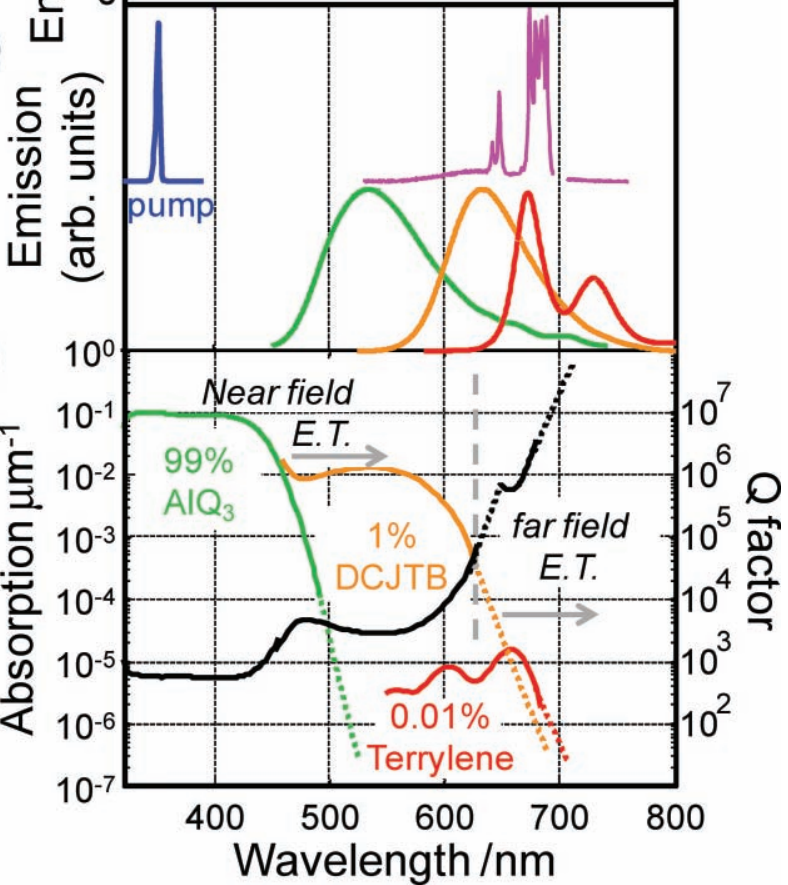

Figure 2. (a) Energy diagram of the cascaded near (dashed) and far (solid) field energy transfer. (b) The photoluminescent spectrum of each dye, with the lasing spectrum shown above. (c) The absorption spectrum of each dye in the laser cavity together with the corresponding $Q$-factor (black). 

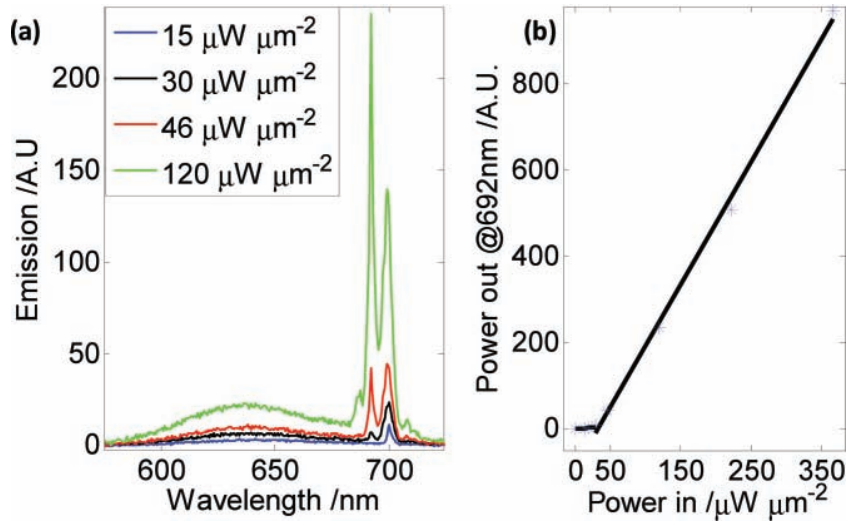

Figure 3. (a) Evolution of the emission spectrum for various pump intensities. (b) Lasing power at $\lambda=692 \mathrm{~nm}$ versus pump power showing lasing power threshold at $30 \mu \mathrm{W} \mu \mathrm{m}^{-2}$.

streak camera pictures is composed from three important wavelength ranges; the short wavelength range $\lambda=630$ $650 \mathrm{~nm}$, which is attributed to DCJTB emission, the long wavelength range $\lambda=670-690 \mathrm{~nm}$, which is attributed to Terrylene emission, and an intermediate range $\lambda=650-670 \mathrm{~nm}$, which can be shared by DCJTB and Terrylene. Analysis of this latter spectral region is complex because both materials could be stimulated by the other's emission. Considering only the pure DCJTB and Terrylene spectral regions, a qualitative analysis of Figure 3 reveals a trend to longer Terrylene transients as the Terrylene concentration is reduced, suggesting an extension in the lifetime of longer wavelength photons in the cavity. In Figure $\mathbf{5}$, we plot a typical DCJTB emission transient at $\lambda=640 \mathrm{~nm}$ and a typical Terrylene emission transient at $\lambda=$ $674 \mathrm{~nm}$ as a function of Terrylene concentration. The entire set of transient data is presented in the Supporting Information.

As expected the Terrylene transients show significant extension in the lifetime of the cavity photons at low Terrylene concentrations. Relative to the transient lifetime for a Terrylene concentration of $0.02 \%$, the lifetime at concentrations of $0.01 \%$ and $0.0075 \%$ is longer by $\Delta \tau=1.45 \mathrm{~ns}$ and $\Delta \tau=1.87 \mathrm{~ns}$, respectively. The corresponding increase in the $Q$-factor, and thus its minimum value is $Q=4.0 \times 10^{6}$ and $Q=5.2 \times 10^{6}$ at Terrylene concentrations of $0.01 \%$ and $0.0075 \%$. In terms of propagation distance, the initial pump photon is absorbed within a submicrometer thickness while the last lasing photon propagates for at least half a meter in the cavity.

This is not the first laser to employ organic dyes and other materials in a low-loss, whispering-gallery resonator. For example, individual highly diluted organic dyes or quantum dots were embedded into high- $Q$ resonators in resonant pumping schema ${ }^{[20]}$ and non-resonant pumping schema. ${ }^{[21-24]}$ In these examples the self absorption of the single gain material sets the gain versus loss balance and thus any increase in the $Q$-factor is associated with lower pump absorption.

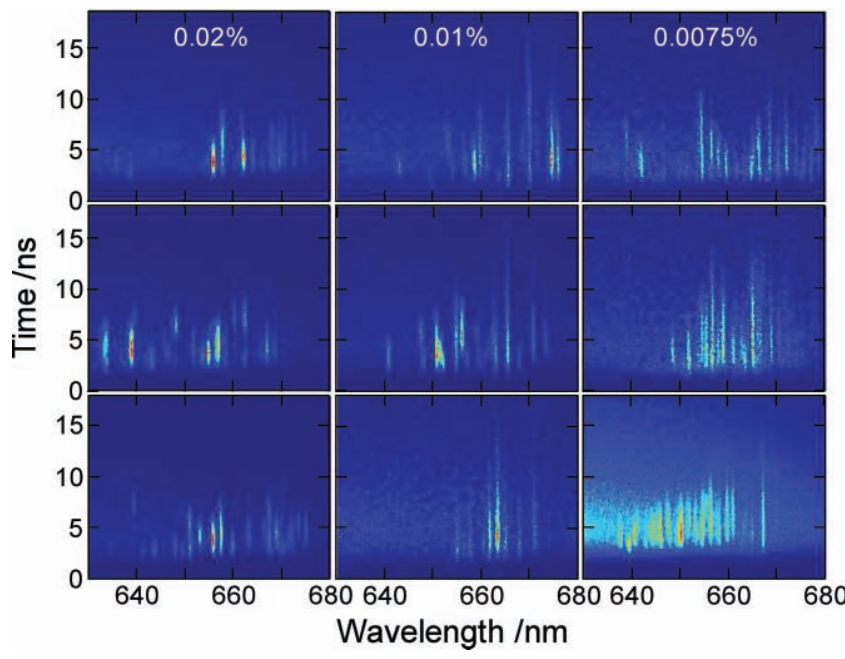

Figure 4. Typical streak camera measurements for various Terrylene concentrations

In another approach, Förster energy transfer was employed between two ${ }^{[25-27]}$ and even more materials, ${ }^{[28]}$ but since the efficiency of Förster energy transfer is dramatically reduced at acceptor concentrations much lower than $1 \%$, the minimum concentration of dyes employed was approximately $1 \%$ and the $Q$-factor obtained was lower than $10^{4} .{ }^{[29]}$ Rather, the combination of high- $Q$ factor and high pump absorption obtained here is due to the combination of a low loss optical cavity and low material absorption losses due to the use of the energy cascade, which starts as non-resonant pumping of a low- $Q$ cavity and ends as resonant pumping of high- $Q$ cavity.

To conclude, we demonstrate a pumping technique for high- $Q$ micro-lasers, based on cascaded energy transfer. The technique significantly increases the efficiency of broad band pumping, thereby overcoming the need for resonantly-matched lasers or precisely aligned III-V semiconductor gain elements to pump high- $Q$ micro-lasers. Consequently, this approach can open the way for fully integrated on-chip ultra-sharp optical sources.

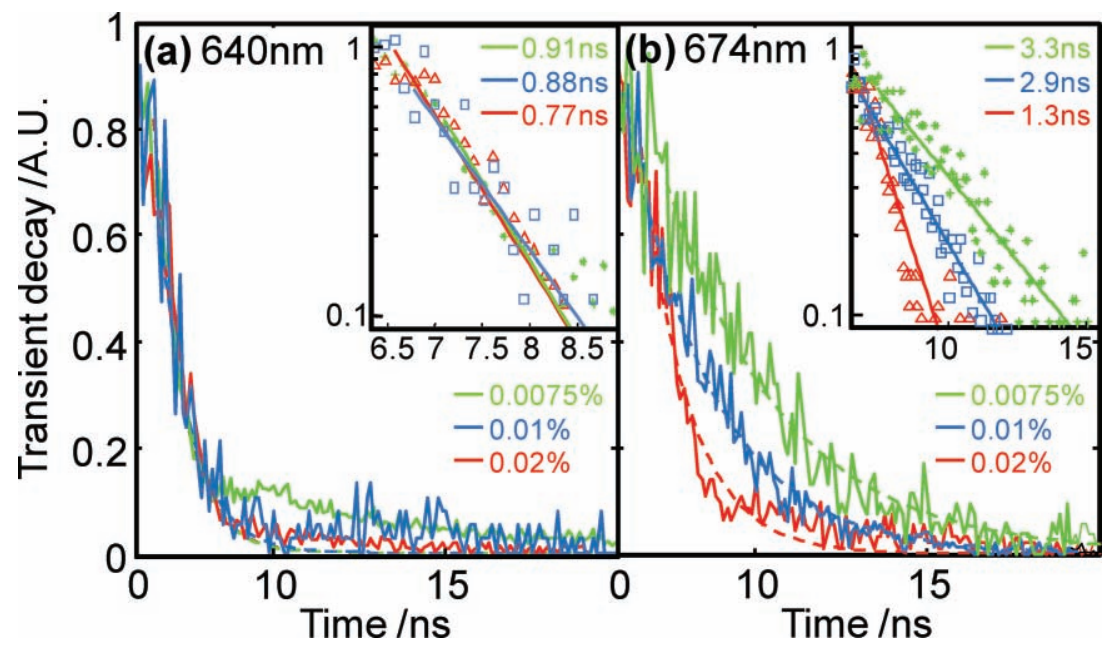

Figure 5. Lifetime measurements of (a) pure DCJTB emission and (b) pure Terrylene emission for various Terrylene concentrations. 


\section{Experimental Section}

The micro-ring cavities employed here are $\mathrm{SiO}_{2}$ on silicon, and possess a large diameter that typically varies between $20 \mu \mathrm{m}$ and $50 \mu \mathrm{m}$ while the small diameter is about $4 \mu \mathrm{m}$. The typical $Q$-factor of the uncoated resonator is $10^{8}$ with a refractive index of 1.455 . To deposit the dyes, the resonators are first spun coated with Terrylene molecules ${ }^{[15]}$ at various concentrations (between $0.0075 \%-0.02 \%$ by weight) in polymethylmethacrylate (PMMA). The thickness of the PMMA layer is estimated to be $100 \mathrm{~nm}$, and the refractive index is 1.49 . Next a $120 \mathrm{~nm}$ thick layer of tris(8-hydroxyquinoline) aluminium (AlQ3) is deposited using thermal evaporation. The AlQ3 is doped with $1 \%$ DCJTB (4-(dicyanomethylene)-2-t-butyl-6-(1,1,7,7-tetramethyljulolidyl-9-enyl)$4 \mathrm{H}$-pyran). The refractive index of the layer is 1.72 . The typical stability of DCJTB under electrical excitation in the controlled environment of a packaged OLED is estimated to be 100 years. ${ }^{[30,31]}$ The simulation of the lasing mode in Figure 1c assumed a resonator with $40 \mu \mathrm{m}$ diameter coated similarly to the experiment. The simulation yielded a confinement factor of no more than $5 \%$ for the AlQ3-DCJTB layer and $15 \%$ for the PMMA-Terrylene layer. The absorption cross-section in Figure $2 \mathrm{c}$ is determined by measuring the absorption of a thick layer and normalizing it to the width of lasing mode $(1 \mu \mathrm{m})$ multiplied by the confinement factor. Energy is applied using a solid state laser with $1.5 \mathrm{~ns}$ pulse width and a $200 \mu \mathrm{m}$ FWHM Gaussian beam at $\lambda=$ $349 \mathrm{~nm}$ incident from above the resonator and not matched to the cavity resonances. The pump Gaussian laser beam at $\lambda=349 \mathrm{~nm}$ is focused to a FWHM spot size of $200 \mu \mathrm{m}$, and attenuated by neutral density filters by 30 to $49 \mathrm{~dB}$ from an initial pulse energy of $144 \mu$ ). For on-chip applications, it may ultimately be more convenient to replace the UV laser with fast response on-chip UV light emitting diode (LED). ${ }^{[32]}$ Scattered light from the resonator is monitored by placing the tip of a multi mode fiber at the side of the resonator. The fiber is coupled to an optical spectrum analyzer. The scattered light includes some of the pump UV light but also photoluminescence and lasing scattered from the cavity. Due to measurement limitations, we were not able to observe the photoluminescence at sub threshold pump powers. The experiments with the Streak camera were done in free space; the scattering from the resonators was collected by a collimating lens onto the slit of the Streak camera.

\section{Supporting Information}

Supporting Information is available from the Wiley Online Library or from the author.

\section{Acknowledgements}

This work was supported as part of the Center for Excitonics, an Energy Frontier Research Center funded by the U.S. Department of Energy, Office of Science, Office of Basic Energy Sciences under Award Number DE-SC0001088 (MIT) and the AFOSR Young Investigator Program, Award Number FA9550-10-1-0078 (Michigan) and the Coherix instant innovation (Michigan). C.R. was also partially supported by Institute for Soldier Nanotechnologies funded under Contract W911NF-07-D-0004 with the U.S. Army Research Office. C.R. would also like to thank Dr. Steven Kooi for his help with the transient measurements

Received: February 4, 2011

Revised: April 8, 2011

Published online: May 24, 2011
[1] C. Y. Chao, W. Fung, L. J. Guo, IEEE J. Selected Top. Quantum Electron. 2006, 12, 134

[2] F. Vollmer, D. Braun, A. Libchaber, M. Khoshsima, I. Teraoka, S. Arnold, Appl. Phys. Lett. 2002, 80, 4057.

[3] A. Serpenguzel, S. Arnold, G. Griffel, Opt. Lett. 1995, 20, 654.

[4] Optical Processes in Microcavities (Eds:R. K. Chang, A. J. Campillo), World Scientific, Singapore, 1996.

[5] F. Treussart, V. S. Ilchenko, J. F. Roch, J. Hare, V. Lefevre-Seguin, J. M. Raimond, S. Haroche, Eur. Phys. J. D 1998, 1, 235.

[6] S. M. Spillane, T. J. Kippenberg, K. J. Vahala, Nature 2002, 415, 621.

[7] B. E. Little, S. T. Chu, P. P. Absil, J. V. Hryniewicz, F. G. Johnson, F. Seiferth, D. Gill, V. Van, O. King, M. Trakalo. IEEE Photon. Technol. Lett. 2004, 16, 2263.

[8] M. Ferrera, L. Razzari, D. Duchesne, R. Morandotti, Z. Yang, M. Liscidini, J. E. Sipe, S. Chu, B. Little, D. J. Moss, Nat. Photonics 2008, 2, 737

[9] A. Yariv, Quantum Electronics, 3rd ed., Wiley (1989).

[10] D. K. Armani, T. J. Kippenberg, S. M. SpillaneK. J. Vahala, Nature 2003, 421, 925.

[11] M. W. Fleming, A. Mooradian, Quant. Elect. 1981, QE-17, 44.

[12] W. Liang, V. S. Ilchenko, A. A. Savchenkov, A. B. Matsko, D. Seidel, L. Maleki, Opt. Lett. 2010, 35, 2822.

[13] T. Carmon, H. Rokhsari, L. Yang, T. J. Kippenberg, K. J. Vahala, Phys. Rev. Lett. 2005, 94, 223902.

[14] M. J. Currie, J. K. Mapel, T. D. Heidel, S. Goffri, M. A. Baldo, Science 2008, 321, 226.

[15] F. Nolde, J. Qu, C. Kohl, N. G. Pschirer, E. Reuther, K. Müllen, Chem. Eur. J. 2005, 11, 3959.

[16] M. Oxborrow, IEEE Transactions On Microwave Theory And Techniques 2007, 55, 1209.

[17] Th. Förster, Discuss. Faraday Soc. 1959, 27, 7

[18] C. W. Tang, S. A. VanSlyke, C. H. Chen, J. Appl. Phys. 1989, 65, 9.

[19] C. H. Chen, C. W. Tang, J. Shi, K. P. Klubeck, Macromol. Symp. 1998, $125,49$.

[20] S. I. Shopova, G. Farca, A. T. Rosenberger, W. M. S. Wickramanayake, N. A. Kotov, Appl. Phys. Lett. 2004, 85, 6101.

[21] B. Min, S. Kim, K. Okamoto, L. Yang, A. Scherer, H. Atwater, K. J. Vahala, Appl. Phys. Lett. 2006, 89(19), 191124.

[22] Y. Kawabe, Ch. Spiegelberg, A. Schulzgen, M. F. Nabor, B. Kippelen, E. A. Mash, P. M. Allemand, M. Kuwata-Gonokami, K. Takeda, N. Peyghambarian, Appl. Phys. Lett. 1998, 72, 141.

[23] S. I. Shopova, H. Zhou, X. Fan, Appl. Phys. Lett. 2007, 90, 221101.

[24] S. Strauf, K. Hennessy, M. T. Rakher, Y. S. Choi, A. Badolato, L. C. Andreani, E. L. Hu, P. M. Petroff, D. Bouwmeester, Phys. Rev. Lett. 2006, 96, 127404.

[25] C. E. Moeller, C. M. Verber, A. H. Adelman,Appl. Phys. Lett. 1971, 18, 278.

[26] M. Berggren, A. Dodabalapur, R. E. Slusher, Appl. Phys. Lett. 1997, $71,2230$.

[27] A. Dodabalapur, M. Berggren, R. E. Slusher, Z. Bao, A. Timko, P. Schiortino, E. Laskowski, H. E. Katz, O. Nalamasu, IEEE J. Selected Top. Quantum Electron. 1998, 4, 67.

[28] M. Berggren, A. Dodabalapur, R. E. Slusher, Z. Bao, Nature 1997, 389,466

[29] M. Koschorreck, R. Gehlhaar, V. G. Lyssenko, M. Swoboda, M. Hoffmann, K. Leo, Appl. Phys. Lett 2005, 87, 181108.

[30] V. V. Jarikov, D. Y. Kondakov, C. T. Brown, J. App. Phys 2007, 102

[31] S. Mais, J. Tittel, T. Basche, C. Bräuchle, W. Göhde, H. Fuchs, G. Müller, K. Müllen, J. Phys. Chem. A. 1997, 101, 8435.

[32] Y. Yang, G. A. Turnbull, I. D. W. Samuel, Appl. Phys. Lett. 2008, 92, 306. 